which a high-pressure water jet struck the abdominal wall at a tangent causing a wound $10-\mathrm{cm}$ long together with surgical emphysema. Recovery was uneventful. My patient's heavy protective clothing decreased the velocity of the jet and dispersed the reduced kinetic energy in the abdominal wall, so that it caused no internal injuries. Since the jet was fired only momentarily the mass of water was minimal.

${ }^{1}$ Neill RWK, George B. Penetrating intra-abdominal injury caused by high-pressure water jet. $B r$ Med $\mathcal{F} 1969$;ii:357-8.

2 Ward Gardner A. High-pressure water injury. Trans Soc Occup Med $1966 ; 16: 30$.

(Accepted 4 March 1980)

Dr Gray's Hospital, Elgin, Morayshire

J L M DE BEAUX, MB, FRCS, consultant surgeon

\section{Inadequate information about drug overdosage in manufacturers' data sheets}

Pharmaceutical companies are required ${ }^{1}$ to supply doctors with data sheets giving information about their drugs, including the likely effects and treatment of overdosage. We reviewed the information about overdosage in the current Data Sheet Compendium. ${ }^{2}$

\section{Analysis of data sheets}

Out of the 1035 data sheets about oral drugs, only $736(71 \%)$ contain information about overdos̄age. Thirty-four of these give some information about the possible effects but no advice about treatment, and $207(28 \%)$ advise about treatment without stating the clinical features of toxicity. Three hundred and forty-seven data sheets concern drugs for injection but 96 $(28 \%)$ contain no information about overdosage. Four hundred and twentynine data sheets concern products for topical use. Most $(72 \%)$ of these give no information about toxicity when taken by mouth, although some products contain potentially dangerous substances, such as salicylates, phenol, or malathion. Nevertheless, a few data sheets give useful information about the likely effects of swallowing ophthalmic ointment (for example, Vira-A Parke Davis) or suppositories (Anusol, Warner), or even intrauterine injection of disinfectant (Cetavlon, ICI). Twenty manufacturers $(16 \%)$ provide no information about overdosage of any of their products.

Unfortunately, the information about overdosage in many data sheets is incomplete, vague, misleading, or wrong, and in some the treatment recommended is frankly dangerous. There are 40 data sheets about products containing paracetamol but five give no information about overdosage and only $21(52 \%)$ mention that liver damage may occur. Effective specific treatment for paracetamol poisoning has been available since $1974^{3}$ but is recommended in only 15 data sheets, whereas 10 advise supportive or symptomatic treatment and three actually state that there is no specific antidote. One data sheet advises alkaline diuresis, which is ineffective and potentially dangerous. Many data sheets advise the use of "antidotes" but do not mention intensive supportive therapy. Noradrenaline or metaraminol are recommended for hypotension, but not sensible measures such as raising the foot of the bed, restoration of plasma volume, and correction of hypoxia. Obsolete drugs recommended for treatment include tannic acid, analeptics (picrotoxin, bemegride, leptazol, amphetamine), and paregoric. Emetics, "universal antidote," subcutaneous pilocarpine, and respiratory stimulants are advised for overdosage of the anti-emetic combination of dicyclomine and doxylamine, but supportive treatment is not discussed. Overdosage of cardiac glycosides may cause severe hyperkalaemia ${ }^{4}$ but the data sheet about lanatoside $C$ advises treatment with potassium chloride. Those for digoxin and medigoxin, however, suggest glucose and insulin for treating hypokalaemia. Fluid restriction is advised for overdosage of the diuretic bendrofluazide and correction of electrolytes with ammonium chloride, potassium chloride, and hypertonic saline. It is implied that artificial respiration may be needed for overdosage of hydrochlorothiazide. Overdosage of pentaerythritol tetranitrate may cause severe headache, which one manufacturer suggests should be treated with caffeine, neostigmine, or amphetamine.

\section{Comment}

Drug overdosage is common and accurate information about the likely consequences and necessary treatment is essential. The Data Sheet Compendium should be a useful source of up-to-date information, but unfortunately some data sheets are inadequate or dangerously wrong. They should contain accurate and relevant advice about the acute toxicity of all products, including topical preparations which are occasionally taken by mouth. It is helpful to know if a product is nontoxic so that unnecessary anxiety and treatment may be avoided. We know the difficulties of providing reliable information about overdosage: we look after poisoned patients ourselves and give advice through the poisons information services. Some drug manufacturers have been concerned and extremely helpful with our inquiries about overdosage of their products. Others, however, have been unwilling or unable to provide useful information, especially about animal toxicity studies of new drugs, and seem to believe that no problem exists. Pharmaceutical companies should supply doctors and the poisons information services with adequate information about their drugs.

${ }^{1}$ Statutory Instrument 1972 No 2076. The medicines (data sheet) regulations 1972. London: HMSO, 1972.

${ }^{2}$ Association of the British Pharmaceutical Industry. Data sheet compendium 1979-80. London: Pharmind Publications, 1979.

${ }^{3}$ Prescott LF. Prevention of hepatic necrosis following paracetamol overdosage. Health Bull (Edinb) 1978 ;36:204-12.

${ }^{4}$ Ekins BR, Watanabe AS. Acute digoxin poisonings: review of therapy. Am f Hosp Pharm 1978;35:268-77.

(Accepted 21 February 1980)

Accident and Emergency Department, General Infirmary, Leeds LS1 3EX

R N ILLINGWORTH, BM, MRCP, senior registrar

Regional Poisoning Treatment Centre and University Department of Therapeutics and Clinical Pharmacology, Royal Infirmary, Edinburgh EH3 9YW

L F PRESCOTT, MD, FRCPED, consultant physician and reader in clinical pharmacology

\section{Late disruption of initially satisfactory stapled anastomoses}

The circular staple gun has been successfully used for colorectal anastomosis ${ }^{1}$ and devascularisation of oesophageal varices. ${ }^{2}$ We have used the American EEA staple gun for 97 intestinal procedures, and we report here four cases of late dehiscence which occurred despite a complete staple ring being seen at radiology in the first week after operation.

\section{Case reports}

Case 1-A 73-year-old woman underwent an anterior resection for diverticular disease under gentamicin and metronidazole prophylaxis with a wellprepared bowel. Intestinal continuity was restored with the American EEA staple gun: two complete rings of intestine were present in the cartridge. On the sixth day after operation a radiograph showed that the staple line was complete. She was discharged on the ninth day after operation but had had a small rectal bleed 24 hours earlier. She was readmitted nine days later with peritonitis. A radiograph showed a break in the staple line, and a barium enema showed a large leak at this site. Dehiscence of the anastomosis with purulent peritonitis and a large pelvic abscess was found at a second laparotomy.

Case 2-A 68-year-old diabetic with a rectal carcinoma had an anterior resection under gentamicin and metronidazole prophylaxis with a satisfactorily prepared colon. Colorectal anastomosis was performeci with the American EEA staple gun. Two complete rings of colon were present in the cartridge. On the eighth day after operation a radiograph showed a complete staple ring. Subsequently the patient developed a rectal haemorrhage and on the 12th day a radiograph showed a break in the staple ring and a watersoluble barium enema showed a small leak at this site.

Case 3-A 66-year-old woman with a rectal carcinoma had an anterior resection using the EEA staple gun under cefoxitin prophylaxis in a wellprepared colon. Two complete intestinal rings were present in the cartridge. A radiograph on the seventh day after operation confirmed a complete staple ring. A contrast study on the 10th day showed a complete staple ring and no anastomotic leak. Postoperative pyrexia persisted until the 17th day when pus discharged per rectum and a radiograph then showed a posterior disruption of the staple ring; pus was seen to discharge from the anastomosis at sigmoidoscopy. 